\title{
Development of a Multiplex-PCR probe system for the proper identification of Klebsiella variicola
}

\author{
Ulises Garza-Ramos*, Jesús Silva-Sánchez ${ }^{1}$, Esperanza Martínez-Romero², Perla Tinoco ${ }^{1}$, Marisol Pina-Gonzales ${ }^{1}$, \\ Humberto Barrios ${ }^{1}$, Jesús Martínez-Barnetche ${ }^{3}$, Rosa Elena Gómez-Barreto ${ }^{3}$ and Juan Tellez-Sosa ${ }^{3}$
}

\begin{abstract}
Background: Klebsiella variicola was very recently described as a new bacterial species and is very closely related to Klebsiella pneumoniae; in fact, $K$. variicola isolates were first identified as $K$. pneumoniae. Therefore, it might be the case that some isolates, which were initially classified as $K$. pneumoniae, are actually $K$. variicola. The aim of this study was to devise a multiplex-PCR probe that can differentiate isolates from these sister species.

Result: This work describes the development of a multiplex-PCR method to identify $K$. variicola. This development was based on sequencing a $K$. variicola clinical isolate (801) and comparing it to other K. variicola and K. pneumoniae genomes. The phylogenetic analysis showed that $K$. variicola isolates form a monophyletic group that is well differentiated from K. pneumoniae. Notably, the isolate K. pneumoniae 342 and K. pneumoniae KP5-1 might have been misclassified because in our analysis, both clustered with $K$. variicola isolates rather than with $K$. pneumoniae. The multiplex-PCR (M-PCR-1 to 3) probe system could identify $K$. variicola with high accuracy using the shared unique genes of $K$. variicola and $K$. pneumoniae genomes, respectively. M-PCR-1 was used to assay a collection of multidrug-resistant (503) and antimicrobial-sensitive (557) K. pneumoniae clinical isolates. We found K. variicola with a prevalence of $2.1 \%$ $(23 / 1,060)$, of them a $56.5 \%(13 / 23)$ of the isolates were multidrug resistant, and $43.5 \%(10 / 23)$ of the isolates were antimicrobial sensitive. The phylogenetic analysis of $r p o B$ of $K$. variicola-positive isolates identified by multiplex-PCR support the correct identification and differentiation of $K$. variicola from $K$. pneumoniae clinical isolates.
\end{abstract}

Conclusions: This multiplex-PCR provides the means to reliably identify and genotype $K$. variicola. This tool could be very helpful for clinical, epidemiological, and population genetics studies of this species. A low but significant prevalence of $K$. variicola isolates was found, implying that misclassification had occurred previously. We believe that our multiplex-PCR assay could be of paramount importance to understand the population dynamics of $K$. variicola in both clinical and environmental settings.

Keywords: Pathogen, Genome comparison, Prevalence, ESBL, Endophytic diazotrophic bacteria, Symbiosis

\section{Background}

The genus Klebsiella belongs to the Enterobacteriaceae family and comprises Gram-negative opportunistic nonmotile pathogens with a mucoid aspect. The species that comprise the genus Klebsiella are ubiquitous in nature and are present in three common habitats: i) the environment, where they can be found in water, soil and plants; ii) the mucous surfaces of mammals, [1] and iii) in symbiosis with insects [2]. In plants, they are endophytic and diazotrophic bacteria; in other words, they

\footnotetext{
* Correspondence: ulises.garza@insp.mx

'Departamento de Diagnóstico Epidemiológico, Av. Universidad \# 655, Col.

Sta. Ma. Ahuacatitlán, C.P. 62100 Cuernavaca, Morelos, Mexico

Full list of author information is available at the end of the article
}

associate with plants by colonizing their internal tissues and providing enough nitrogen that the hosts mitigate their nitrogen deficiency [3]. In mammals, these bacteria colonize the upper respiratory tract and the gastrointestinal tract. Most Klebsiella-related infections are associated with hospitalization. These pathogens are the causative agents of 7 to $10 \%$ of all nosocomial infections reported in Europe, Latin-America and North America [1]. $K$. variicola was described as a new bacterial species in 2004 [4] based on the phylogenetic analysis of six housekeeping (rpoB, gyrA, nifH, infB, phoE and $m d h$ ) genes and DNA-DNA hybridizations. This work identified that $8 \%$ of the K. pneumoniae isolates corresponded to $K$. variicola, in both clinical isolates and environmental 
isolates [4]. Subsequently, multidrug-resistance phenotypes and extended-spectrum $\beta$-lactamase (ESBL) producers were identified for $K$. variicola during an intra-hospital outbreak at a pediatric hospital in Mexico $[4,5]$. Additionally, this species has been recently reported as a symbiotic nitrogen-fixing bacteria and has frequently been isolated from the fungus gardens of leaf-cutter ant colonies collected in Central and South America [2]. To date, the correct identification of Klebsiella species has not been easily achieved in microbiological laboratories because several species of this genus share similar biochemical profiles. Commercial kits, both manual and automated, do not include some of the organisms in their databases or the necessary substrates to differentiate species [6]. Some studies have been expressly designed to identify biochemical markers that can differentiate $K$. variicola from $K$. pneumoniae. For instance, the inability to ferment adonitol, which was one of the characteristics used by Rosenblueth et al. [4]. A recent study rejected this proposal because it was determined that this biochemical characteristic is variable among different isolates. Currently, the differentiation of $K$. variicola and $K$. pneumoniae using biochemical tests is not possible [7]. To date, $K$. variicola is one of the species of the genus Klebsiella that is not even included in commercial kits, and it has been described in several environments as a pathogen in humans (causing nosocomial infections), a symbiont in insects [2] and an endophyte in plants [4]. This lack of microbiology and molecular tools to differentiate these bacterial species may be reflected in misidentification, such as the case of K. pneumoniae 342, an endophyte in plants [3], which has been recently included within the cluster of the $K$. variicola species $[8,9]$.

We describe the development of a multiplex-PCR method to identify $K$. variicola; notably, this development was based on sequencing a $K$. variicola clinical isolate (801) and comparing it to other K. variicola and K. pneumoniae genomes. To complement this, we conducted an array of phylogenetic analyses of selected loci that clearly differentiate $K$. variicola from $K$. pneumoniae. Furthermore, we describe the prevalence and characteristics of $K$. variicola among $K$. pneumoniae isolates (both multidrug resistant and sensitive) obtained from Mexican hospitals.

\section{Methods}

\section{K. variicola 801 clinical isolate and genome sequencing}

The $K$. variicola 801 isolate was obtained from the blood of a newborn with pneumonia at the Children's Hospital in Tabasco, Mexico in 1996. This isolate, together with ten additional isolates (803-812), belongs to an intrahospital outbreak identified at this hospital [5]. The $K$. variicola 801 clinical isolate was selected for genome sequencing based on the phylogenetic analysis of six housekeeping (rpoB, gyrA, nifH, infB, phoE and $m d h$ ) [4]. The genome was assembled and annotated using the bioinformatics software Maq (Mapping assembly) and MicroScope platform (https://www.genoscope.cns.fr/agc/ microscope/home/index.php), respectively.

\section{Ethics statement}

This project was exempt from review by the Ethic Commission at INSP because it does not involve human subjects and/or it is not an academic study and/or it does not include the analysis of data previously obtained from another study requiring the patients' informed consent. On the other hand, the bacteria included in the study were obtained by routine procedures in each of the hospitals involved.

\section{Phylogenetic analyses of housekeeping genes}

The nucleotide sequences of five housekeeping genes, gyrA, phoE, infB, $m d h$ and rpoB, were obtained from K. variicola 801, K. variicola At-22, K. pneumoniae 342, K. variicola BZ19, K. variicola CAG:634, K. pneumoniae KP5-1, K. pneumoniae MGH78578, K. pneumoniae NTUH-K2044, K. pneumoniae KTCT 2242, K. oxytoca KTCT 1686, Salmonella enterica Ty21a, Escherichia coli K12 MG1655 genomes. Using these concatenate sequences of the five housekeeping genes, a maximumlikelihood phylogeny was generated using Mega v5.05 [10], with a Tamura-Nei model and 1,000 bootstrap replications.

\section{Comparison and functional category of shared unique proteins of $K$. variicola and $K$. pneumoniae genomes}

The aim of this analysis was to identify the shared unique proteins of $K$. variicola $\left(\mathrm{Kv} \_801, \mathrm{Kv} \_\mathrm{At}-22\right.$ and Kp_342 [available at the moment of analysis]) and $K$. pneumoniae (Kp_NTUHK2044 and Kp_MGH78578) genomes. The predicted proteomes of K. variicola 801 (Kv_801) [5,246 proteins], K. variicola At-22 (Kv_At22) [5,057 proteins], K. pneumoniae 342 (Kp_342) [5,766 proteins], K. pneumoniae MGH78578 (Kp_MGH78578) [5,184 proteins] and NTUH-K2044 (Kp_NTUH-K2044) [5,262 proteins] were considered for comparisons. All of the comparisons were carried out using the BLASTp program with default values [11] (considers sequences as homologous if they have approximately $40 \%$ amino acid identity and $70 \%$ amino acid sequence coverage). For determining the shared unique proteins of $K$. variicola, we proceeded as follows: first, $\mathrm{Kv} \_801$ and $\mathrm{Kv} \_$At-22 were compared, and the set of shared proteins found in this comparison was then compared with Kp_342 to identify the shared proteins among the $K$. variicola genomes. Subsequently, the shared proteins among the $K$. variicola genomes were compared with the $K$. pneumoniae proteomes (Kp_MGH78578 and Kp_NTUH-K2044) to 
define the unique proteins sequences of $K$. variicola. A similar logic was used to define the proteins unique to K. pneumoniae; briefly, Kp_MGH78578 and Kp_NTUHK2044 were compared, and the sequences shared by both were then compared against the $K$. variicola genomes (Kv_801, Kp_342 and Kv_At-22), thus identifying the shared unique protein sequences of $K$. pneumoniae. Next, we used the megablast option, which was designed for comparisons of highly similar sequences (percent identity $\geq 95 \%$ ), to rule out that proteins unique to either K. variicola At-22 or $K$. pneumoniae 342 could be present in other related bacterial species. The functional category of the unique proteins identified in the K. pneumoniae and $K$. variicola genomes was determined using the BLASTp program [11]. (All non-redundant GenBank CDS translations + PDB + SwissProt + PIR + PRF excluding environmental samples from WGS projects). The proteins involved in the horizontal transfer of genetic material or those present in other organisms were eliminated. The proteins with a known or unknown (putative) function unique to $K$. pneumoniae and $K$. variicola were saved.

\section{Design of oligonucleotides, $m$ tn $C$ gene analysis and conditions for single- and multiplex-PCR}

Oligonucleotides specific for shared unique genes identified in $K$. variicola and $K$. pneumoniae were designed using a similar melting temperature $(\mathrm{mT})$, between $58^{\circ} \mathrm{C}$ and $62^{\circ} \mathrm{C}$, and amplifying different amplicon lengths (from 340- to 888 -bp). The analysis of the $m t n C$ gene code for a protein specific to the genus Klebsiella spp $[12,13]$ was used as a molecular marker of Klebsiella genera. Nucleotide analysis by BLASTn of the $m t n C$ gene was carried out, and the alignment considered the gene sequences corresponding to $K$. pneumoniae NTUH-K2044 (GenBank ID: AP006725.1), K. pneumoniae MGH78578 (GenBank ID: CP000647.1), K. pneumoniae 342 (GenBank ID: CP000964.1), K. variicola At22 (GenBank ID: CP001891.1) and K. oxytoca (GenBank ID: U00148.1). Multiple alignment from these sequences was performed using MEGA 5 [10]. Subsequently, by determining in vitro that the designed oligonucleotides are specific to $K$. variicola, K. pneumoniae and Klebsiella spp., the selected genes were amplified by single PCR using standard conditions. DNA from control strains such as $K$. pneumoniae ATCC 13883, K. variicola ATCC BAA-830 T (CFNE 2004 T), R. terrigena ATCC 33257, R. planticola ATCC 33531, and K. oxytoca ATCC 49134 were used. DNA was extracted using thermal shock, and by boiling and freezing at $96^{\circ} \mathrm{C}$ and $4{ }^{\circ} \mathrm{C}$, respectively. The $r p o B$ gene was included as an amplification control for single PCR using the CM7 [4] and rpoB-M (5' GAGTCAACGGCAACAGCACG3') oligonucleotides.
The conditions for the multiplex amplification of the shared genes unique to $K$. variicola, $K$. pneumoniae and the genus Klebsiella $(m+n C)$ were determined. The following oligonucleotides and concentrations were proposed (Table 1): KV770-F and -R, KP888-F and -R and KmtnC-F and -R (named M-PCR-1), KV1615-F and -R, $\mathrm{KP} 878-\mathrm{F}$ and $-\mathrm{R}$ and $\mathrm{KmtnC}-\mathrm{F}$ and $-\mathrm{R}$ (named M-PCR2) (5 pmol/reaction of $K$. variicola and Klebsiella spp; 25 $\mathrm{pmol} /$ reaction of $K$. pneumoniae). In the combination of KV1000-F and -R, KP888-F and -R and KmtnC-F and -R (named M-PCR-3), the oligonucleotide concentrations were $5 \mathrm{pmol} /$ reaction of $K$. pneumoniae, $1 \mathrm{pmol} /$ reaction of $K$. variicola, and $0.2 \mathrm{pmol} /$ reaction of the genus Klebsiella, respectively (Table 1 ). The amplified fragments underwent electrophoresis in a $1.2 \%$ agarose gel at $100 \mathrm{~V}$ for one hour in $1 \times$ TAE buffer solution ( $40 \mathrm{mM}$ Tris$\mathrm{HCl}, 2 \mathrm{mM}$ acetic acid, $1 \mathrm{mM}$ EDTA); the gel was dyed with ethidium bromide $(5 \mu \mathrm{g} / \mathrm{ml})$. The PCR products were purified using the High Pure PCR Product Purification kit (Roche) following the manufacturer's recommendations. Thereafter, the amplified products were sequenced using the ABI PRISM 3100 system (PerkinElmer Division; Applied Biosystems), using the Sanger method with a Big-Dye Terminator Kit. The sequences were analyzed by BLASTn (http://blast.ncbi.nlm.nih.gov/Blast.cgi).

\section{Screening of $K$. variicola among K. pneumoniae clinical isolates}

Using the multiplex-PCR development in the present work (Table 1), K. variicola was screened using M-PCR1 (Table 1) among 1,331 clinical isolates previously identified as $K$. pneumoniae. The isolates were obtained from 28 different hospitals in Mexico (including pediatric and general hospitals) and included a period between 1990 and 2013 (Additional file 1). For the study, 1,060 isolates were included (according to the PFGE pattern and hospital origin); they comprised $79.6 \%$ of the total and were identified using the API 20E system (Clinical Diagnostic, BioMérieux). In this strain collection, $47.4 \%$ (503) corresponded to multidrug-resistant and ESBL-producing isolates, and $52.6 \%$ (557) corresponded to isolates that were susceptible to most antibiotics (non-ESBL-producer). The latter isolates corresponded only to two hospitals (Additional file 1). All of the isolates obtained from the K. variicola outbreak at a pediatric hospital in Tabasco [5] and the $K$. variicola endophytic isolates obtained from maize $(n=3)$, sugar cane stem $(T 29 A)$, rice roots (CFNE 2006), banana soot, leaves and stem (F2R9 (ATCC BAA-830 T), 6A2 and VI, respectively were included in the study. The latter isolate was previously isolated and characterized by Rosenblueth et al. [4]. The $K$. variicola-positive isolates were confirmed using $\mathrm{M}$ PCR-2 and M-PCR-3. Initially, the DNA of three clinical 
Table 1 Amplification conditions, oligonucleotide combinations, sequence and amplification fragment of multiplex-PCR for $K$. variicola identification

\begin{tabular}{|c|c|c|c|c|c|c|c|}
\hline \multirow{2}{*}{$\begin{array}{l}\text { Amplification } \\
\text { conditions }^{\mathrm{a}}\end{array}$} & \multirow{2}{*}{$\begin{array}{l}\text { Name of } \\
\text { combination } \\
\text { primers }\end{array}$} & \multicolumn{6}{|c|}{ Shared unique genes, oligonucleotides and sequence $\left(5^{\prime}-3^{\prime}\right)$ of each bacterial specie } \\
\hline & & K. pneumoniae & $\begin{array}{l}\text { Amplification } \\
\text { fragment (bp) }\end{array}$ & K. variicola & $\begin{array}{l}\text { Amplification } \\
\text { fragment (bp) }\end{array}$ & Klebsiella spp. & $\begin{array}{l}\text { Amplification } \\
\text { fragment (bp) }\end{array}$ \\
\hline & & phosphohydrolase & & phosphoglycerate mutase & & $\begin{array}{l}\text { phosphopentane phosphatase } \\
\text { (mtnC) }\end{array}$ & \\
\hline \multirow[t]{3}{*}{1} & M-PCR-1 & $\begin{array}{l}\text { KP888-F: } \\
\text { AAGCAAGCCAGAACAGAAAG }\end{array}$ & 888 & $\begin{array}{l}\text { KV770-F: TCCCGAGGTTCA } \\
\text { CATTTCC }\end{array}$ & 449 & $\begin{array}{l}\text { KmtnC-F: CCGCCGACCTTATC } \\
\text { ACTAC }\end{array}$ & 340 \\
\hline & & $\begin{array}{l}\text { KP888-R: } \\
\text { ACTTCGGTTTATCCAGGTC }\end{array}$ & & $\begin{array}{l}\text { KV770-R: AGCGGGTGAAC } \\
\text { GTCGATAC }\end{array}$ & & $\begin{array}{l}\text { KmtnC-R: AGCGGGTGAACGT } \\
\text { CGATAC }\end{array}$ & \\
\hline & & transferase (yphG) & & N-acetyltransferase & & $\begin{array}{l}\text { phosphopentane phosphatase } \\
\text { (mtnC) }\end{array}$ & \\
\hline \multirow[t]{3}{*}{1} & M-PCR-2 & $\begin{array}{l}\text { KP878-F: } \\
\text { ACCGATAACCAGCCTGACTT }\end{array}$ & 878 & $\begin{array}{l}\text { KV1615-F: ACACAACATTT } \\
\text { CAGGCGGCT }\end{array}$ & 499 & $\begin{array}{l}\text { KmtnC-F: CCGCCGACCTTATC } \\
\text { ACTAC }\end{array}$ & 340 \\
\hline & & $\begin{array}{l}\text { KP878-R: CTTTCTTCTGCCCA } \\
\text { CTGTTG }\end{array}$ & & $\begin{array}{l}\text { KV1615-R: GGGCGTGGCTT } \\
\text { TITCATCG }\end{array}$ & & $\begin{array}{l}\text { KmtnC-R: AGCGGGTGAACGT } \\
\text { CGATAC }\end{array}$ & \\
\hline & & phosphohydrolase & & thiopurine S-methyltransferase & & $\begin{array}{l}\text { phosphopentane phosphatase } \\
\text { (mtnC) }\end{array}$ & \\
\hline \multirow[t]{2}{*}{2} & M-PCR-3 & $\begin{array}{l}\text { KP888-F: AAGCAAGCCAGA } \\
\text { ACAGAAAG }\end{array}$ & 888 & $\begin{array}{l}\text { KV1000-F: CTGGGATGTGG } \\
\text { CAATGGTG }\end{array}$ & 438 & $\begin{array}{l}\text { KmtnC-F: CCGCCGACCTTAT } \\
\text { CACTAC }\end{array}$ & 340 \\
\hline & & $\begin{array}{l}\text { KP888-R: ACTTCGGTTTTAT } \\
\text { CCAGGTC }\end{array}$ & & $\begin{array}{l}\text { KV1000-F: AAACTGCGCCT } \\
\text { GCTGTATC }\end{array}$ & & $\begin{array}{l}\text { KmtnC-R: AGCGGGTGAACGT } \\
\text { CGATAC }\end{array}$ & \\
\hline
\end{tabular}

${ }^{\mathrm{a}}$ Multiplex-PCR conditions used under the oligonucleotides combinations. 1: 5pmol/reaction of $K$. variicola and Klebsiella spp, $25 \mathrm{pmol} /$ reaction of $K$. pneumoniae; $2: 25 \mathrm{pmol} /$ reaction of $K$. pneumoniae, 5 pmol/reaction of K. variicola and $1 \mathrm{pmol} /$ reaction of Klebsiella spp. 
isolates was mixed, and the mixtures that were $K$. variicola-positive were assayed subsequently by individual multiplex-PCR.

\section{Phylogenetic analysis and characterization of K. variicola-positive isolates}

The clinical and environmental isolates identified as $K$. variicola (22/30) and $21 \mathrm{~K}$. pneumoniae clinical isolates were selected for the PCR amplification of rpoB genes, using the oligonucleotides from $K$. pneumoniae MLST [14]. Regarding $K$. pneumoniae, isolates were selected from different hospitals (Additional file 1). The PCR products were purified using the High Pure PCR Product Purification kit (Roche) following the manufacturer's recommendations. Thereafter, the amplified products were sequenced in the ABI PRISMA 3100 system (Perkin-Elmer Division; Applied Biosystems) using the Sanger method with the Big-Dye Terminator Kit. The single-gene comparison for the rроB DNA sequence was performed using Mega v5.05 [10]. The rpoB partial nucleotide sequence (501 bases) phylogeny was constructed using the maximum-likelihood method with a Tamura-Nei-parameter model and 100 bootstrap replications. Additionally, the chromosomal $\beta$-lactamase gene from the $K$. variicola 801 isolate was analyzed against the chromosomal encoded $\beta$-lactamases from $K$. variicola At-22, K. variicola CAG:634, K. variicola BZ19, K. pneumoniae 342 and $K$. pneumoniae KP5-1, K. pneumoniae MGH78578 and K. pneumoniae NTUH-K2044 isolates. LEN-type amino acid sequences were obtained from Pasteur Institute Home page (http://bigsdb.web.pasteur.fr/ perl/bigsdb/bigsdb.pl?db=pubmlst_klebsiella_seqdef_public\&page $=$ downloadAlleles) and were included in the analysis from the 17- to 278-amino acid sequence (LEN-6 and LEN-14 alleles were excluded due to the partial sequence). The single-gene comparison for the LEN-alleles amino acid sequence was performed using Mega v5.05 [10]. The phylogeny was constructed using the maximum-likelihood method with a Jones-Taylor-Thornton-parameter model and 100 bootstrap replications.

In all $K$ variicola isolates, the antimicrobial susceptibility against amoxicillin, AMX; cephalothin, CF; cefoxitin, FOX; cefuroxime, CXM; piperaciline, PIP; piperaciline/ tazobactam, TZP; aztreonam, ATM; cefotaxime, CTX; ceftazidime, CAZ, gentamicin, GM; amikacin, AMK; ticarcillin, TIC; tetracycline, TET; nalidixic Acid, NAL; ciprofloxacine, CIP; levofloxacin, LUX and ESBLproduction, respectively, were determined by disk diffusion and the combination disk method, with ceftazidime and cefotaxime applied individually and in combination with clavulanic acid, following the recommendations of the Clinical and Laboratory Standards Institute (CLSI) (M100-S21) [15]. In the first case, the results were interpreted as resistant $R$, intermediate I and susceptible $\mathrm{S}$, according to the CLSI performance standard M100-S21.[15] E. coli ATCC 25922 was used as a reference strain for susceptibility testing. The CTX-M- and SHV-type ESBL genes were screened by PCR using specific primers [16]. The relationship of $K$. variicola isolates was determined by pulse-field gel electrophoresis (PFGE) [17] and interpreted by GelCompar II software (Applied Math, Kortrijk, Belgium). The similarity percentage was represented using a dendrogram derived from UPGMA and Dice coefficients (the band position tolerance and optimization were set at $1.0 \%$ and $1.0 \%$, respectively). In addition, KPC-type-producing $K$. pneumoniae clinical isolates that had previously been described in Mexico $[18,19]$ were included in the fingerprinting analysis.

\section{Results and Discussion}

\section{K. variicola genome characteristics}

The $K$. variicola 801 clinical isolate was pyrosequenced on a 454 Roche FLX TITANIUM platform; de novo assembly was conducted, revealing 91 contigs with an N50 of $131,409 \mathrm{pb}$; the estimated genome size is $5,790,474$ bp with $15 \times$ coverage, and $99.82 \%$ of the bp were above Q40. Genome annotation identified a total of number of genomic objects (CDS, fCDS, rRNA, tRNA, miscRNA) of 5,736 and a number of CDS (total) of 5,620. The annotated genome sequence was deposited in the European Nucleotide Archive under accession number: CDMV01000001. In the present work, the genome of this $K$. variicola 801 clinical isolate was compared with another $K$. variicola and $K$. pneumoniae genomes. Based on those comparisons, a phylogenetic and comparative genomic analysis developed a multiplex-PCR probe system for the proper identification of $K$. variicola. Next, we used this method to evaluate the prevalence of $K$. variicola in a collection of isolates that had previously been identified by biochemical tests as K. pneumoniae.

\section{Phylogenetic analysis of $K$. variicola and $K$. pneumoniae isolates}

$K$. variicola and $K$. pneumoniae isolates, either environmental or clinical, have very similar phenotypic and biochemical characteristics. Thus, their differentiation through conventional or automated biochemical methods is not currently possible. Misclassification of Klebsiella spp. isolates could occur. With this view, a phylogenetic analysis of housekeeping genes was carried out from $K$. variicola and K. pneumoniae isolates. Figure 1 shows that $K$. variicola isolates and K. pneumoniae 342 clustered together to the exclusion of all of the K. pneumoniae isolates. There is full support, with a bootstrap value of 1,000 for the group composed of the $K$. variicola isolates and K. pneumoniae 342. The K. pneumoniae 342 endophytic strain clearly is a strain of $K$. variicola; this assumption was hypothesized when $K$. variicola was 


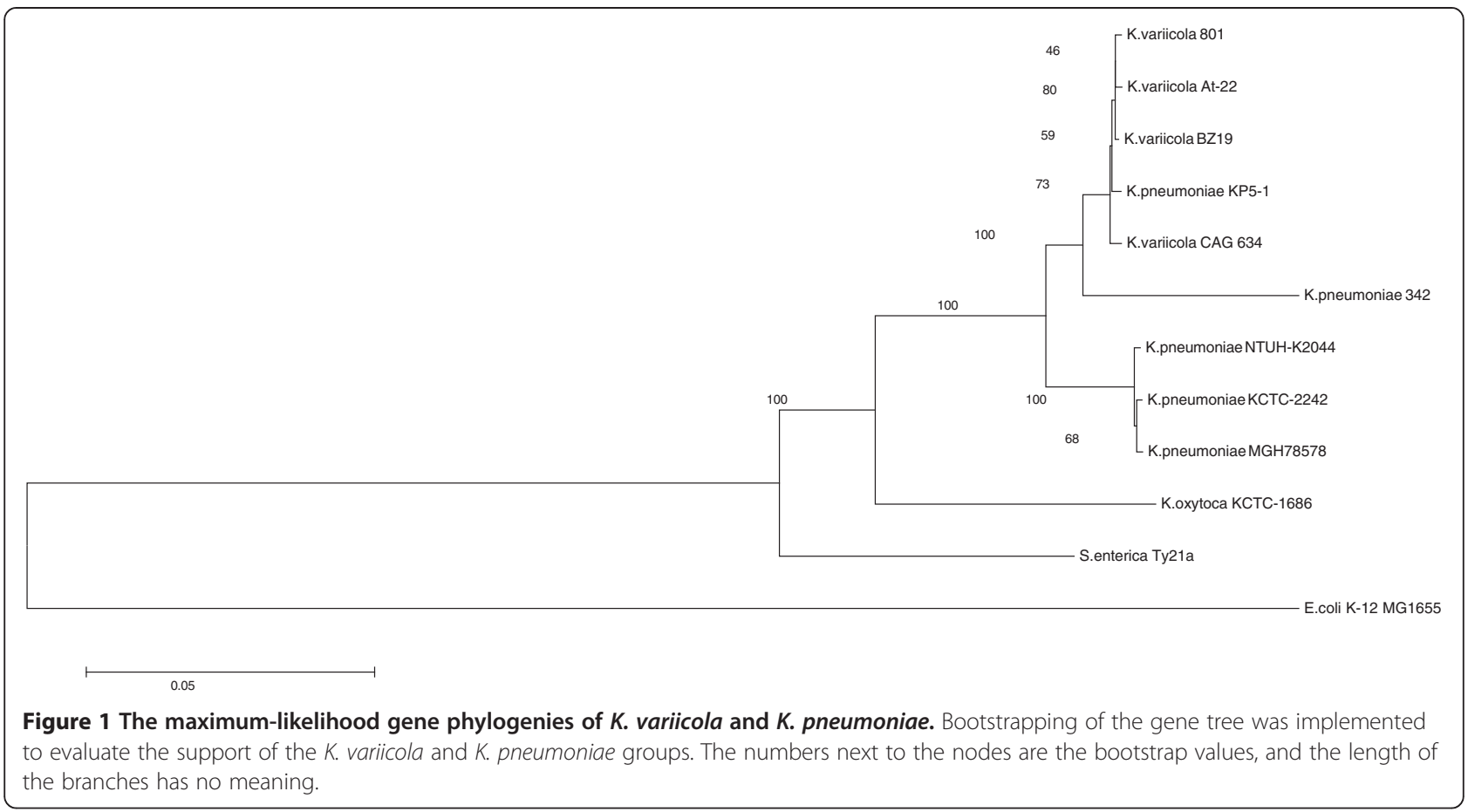

described as a new bacterial species [4] and was recently confirmed in other studies $[9,20,21]$. Thus, in the present study, this bacterium was considered to belong to the $K$. variicola species for the rest of the analysis.

In the seminal work that describes $K$. variicola, one of the defining characteristics of this species is its ability to fix nitrogen. Considering this characteristic, we checked for the presence of the nifH gene, which is essential for nitrogen fixation. The nifH gene was not identified in K. pneumoniae genomes (MGH78578, NTUH-K2004, KTCT 2242 and JM45). Nevertheless, BLASTn analysis of the nifH gene showed a match with different bacterial genera: $K$. variicola At-22, K. variicola CAG:634, K. variicola BZ19, K. pneumoniae 342, K. pneumoniae KP5-1, K. pneumoniae (X13303.1 and J01740.1), K. oxytoca (KTCT 1686), Pantoea sp. (At-9b), Enterobacter sp. (R4-368), R. aquatilis ATCC33071 and P. stutzeri (DSM4166) (data not showed). nifH genes belong to operon nif, which contains a 20-nif gene cluster involved in nitrogen fixation [22]. Historically, the nif gene cluster has been extensively characterized in K. pneumoniae [23]. However, Hazen et al. (2014) described the absence of the nif gene cluster in eight K. pneumoniae NTUH-K2044, MGH 78578, 1162281, JH1, MS 92-3, 1191100241, ATCC13884 and KCTC 2242 clinical isolates [8]. The ability of nitrogen fixation of the $K$. variicola 801 clinical isolate was described by Rosenblueth et al. (2004) [4]. Here, we confirm that this $K$. variicola clinical isolate contains niff-nifQ cluster genes (20 genes), with a $>98$ nucleotide identity with $K$. variicola At-22 and K. pneumoniae 342 nif cluster genes (data not showed). This analysis showed that the nifH gene is a clear characteristic of $K$. variicola isolates, and $K$. pneumoniae isolates that contain the nifH gene correspond rather to $K$. variicola species. As in the case of K. pneumoniae KP5-1 (CP008700), what was recently submitted to GenBank (04-JUN-2014) (see below).

A maximum likelihood phylogeny of LEN-type $\beta$ lactamase, chromosomal from $K$. variicola 801, K. variicola At-22, K. variicola CAG:634, K. variicola BZ19, K. pneumoniae 342 and $K$. pneumoniae KP5-1 genomes was constructed (Additional file 2). The K. variicola 801 isolates showed a new LEN-type allele, LEN-32 with one new amino acid change in the position $68(\mathrm{~S} \rightarrow \mathrm{M})$, which was deposited in the PasteurMLST data base (http:// bigsdb.web.pasteur.fr/perl/bigsdb/bigsdb.pl?db= pubmlst_ klebsiella_seqdef_public\&page $=$ downloadAlleles). LEN-13 was identified in $K$. variicola At-22 and $K$. variicola BZ19. LEN-10, LEN-16 and LEN-28 alleles were identified in K. pneumoniae CAG:634, K. pneumoniae KP5-1 and K. pneumoniae 342, respectively. Duplication in tandem of the LEN-28 gene with 14 other chromosomal genes (lysR-Deor) is contained in the K. pneumoniae 342 chromosome (data not shown). In the case of the $K$. pneumoniae MGH78578 and K. pneumoniae NTUHK2004, the genomes have a different chromosomal $\beta$ lactamase, namely SHV-11, which is closely related to the LEN-type (Additional file 2). These chromosomally encoded $\beta$-lactamases are characteristic of $K$. variicola and $K$. pneumoniae species. They could not be used as 
molecular markers because the SHV-type $\beta$-lactamases have been broadly described for conjugative and nonconjugative plasmids in both $K$. variicola and $K$. pneumoniae isolates [5]. The LEN-type $\beta$-lactamases have not been described on plasmids. Furthermore, LEN-26 is chromosomally encoded in Klebsiella sp. 10982, which is a bacterium recently described as a phylogenetic and metabolic intermediate between the $K$. variicola endophyte and K. pneumoniae clinical isolates [8].

\section{Comparative analysis of the $K$. variicola and $K$. pneumoniae genomes}

Very little is known concerning $K$. variicola or the role that it plays in the environment and hospitals. K. variicola was identified using molecular strategies based on phylogenetic analyses of housekeeping genes [4]. However, previously, analyzing $K$. pneumoniae clinical samples, Brisse and collaborators carried out phylogenetic analyses of gyrA and parC genes and the adonitol fermentation test [24]. They identified three different phylogenetic groups called KpI, KpII and KpIII with KpI being the largest. Group KpIII very likely belongs to $K$. variicola because it has gyrA sequences that are very similar to those found in $K$. variicola [4]. It is noteworthy that although there are differences between these groups, the phenotypic characteristics of $K$. pneumoniae do not change; it is only possible to differentiate these groups using gene sequencing. Comparative genome studies on Klebsiella spp. have been carried out [3,9,13], and the development of biochemical techniques to differentiate $K$. variicola from $K$. pneumoniae has also been tried. For example, Alves et al. [7] tried to implement a biochemical test that includes the adonitol-as-a-carbonsource test as a potential strategy to identify $K$. variicola among $K$. pneumoniae isolates. The adonitol test was found to be variable in both species and, therefore, not reliable to discriminate between these species. To date, a biochemical test to identify $K$. variicola has not yet been determined. Nonetheless, a study carried out by VanVeen et al. [25] reported the identification of bacterial genus and species using mass spectrometry. The study comprised non-fermentative Gram-negative microorganisms, yeast and Enterobacteriaceae, including K. pneumoniae and $K$. variicola. This tool showed the correct identification of the Klebsiella genus, but an incorrect identification of species (minor error) was revealed regarding $K$. variicola in $K$. pneumoniae. Although this molecular technique is highly specific, it still has an error margin and thus cannot be used to identify $K$. variicola. The problems in identifying species within a bacterial genus are not specific to $K$. pneumoniae and $K$. variicola. Efforts to optimize the identification methods of highly related species of other microorganisms have also been tried out. Some tests that have been developed to identify species rely on molecular tools, as in the case of the genus Salmonella [26,27]. A different approach was addressed in this work, with a comparative analysis of the $K$. variicola and $K$. pneumoniae genomes (see methods). This comparison revealed 114 proteins shared by the $K$. variicola genomes but not present in K. pneumoniae. In the case of $K$. pneumoniae genomes, we identified 54 proteins that were shared but not present in $K$. variicola (Additional file 3 ). Excluding the genes involved in horizontal transfer, we identified 79 proteins shared by $K$. variicola and 40 proteins shared by $K$. pneumoniae. Of the proteins that were found to be unique to each species, those involved in metabolism and cellular structure were selected. Therefore, 20 metabolic proteins and 12 structural proteins were selected for $K$. variicola, and four metabolic proteins and six structural ones were selected for K. pneumoniae (Additional file 4). Next, we ruled out the gene sequences of the shared proteins unique to either $K$. variicola At-22 or K. pneumoniae 342 that are present in other related bacterial species (see methods). Therefore, the sequences selected were those exclusively present in the $K$. variicola At-22 and K. pneumoniae 342 genomes or the K. pneumoniae MGH78578 and NTUH-K2044 genomes. These were the three shared metabolic genes unique to $K$. variicola and two shared metabolic genes unique to $K$. pneumoniae. The specific oligonucleotides for the PCR amplification of shared unique genes were designed (Table 1).

\section{Characteristics of multiplex-PCR for $K$. variicola identification}

The inclusion of a PCR amplification control that was also a gene characteristic of Klebsiella genera was considered, and the $m t n C$ gene was selected. The phosphopentane phosphatase $(m t n C$ gene) protein is part of the methionine salvage pathway, which is frequent in bacteria [12]. A recent study reported that the $m t n C$ gene is only contained in $K$. pneumoniae [13]. We carried out a BLASTn (considering the Nucleotide collection [nr/nt] from NCBI database) search using as a query the nucleotide sequence of the $m t n C$ gene taken from the genome of $K$. pneumoniae NTUHK2044. The analysis revealed 22 similar gene sequences in the GenBank, seven of which were bacteria of the genus Klebsiella and showed an identity $>95 \%$. The closest match after those was the sequence of the $m t n C$ gene corresponding to Pantoea vagans (49\%). The $m t n C$ gene from the $K$. pneumoniae NTUH-K2044, K. pneumoniae MGH78578, K. pneumoniae 342 and $K$. variicola At-22 genomes showed a $100 \%$ identity, followed by a $98 \%$ and a $96 \%$ identity from the Klebsiella oxytoca and Enterobacter cloacae ATCC 13047 and Enterobacter sp. 638 genomes, respectively. The design of the oligonucleotides of the $m t n C$ gene from the genus Klebsiella was determined by selecting a fragment in 
which the sequences of $K$. pneumoniae NTUH-K2044, K. pneumoniae MGH78578, K. pneumoniae 342 and $K$. variicola At-22 isolates were identical. The oligonucleotides were named $\mathrm{KmtnC}-\mathrm{F}$ and $\mathrm{KmtnC}-\mathrm{R}$ and amplified a 340-bp fragment (Table 1).

The oligonucleotides designed from the shared unique genes for $K$. variicola were as follows: KV770-F and KV770-R that amplify a 449-bp fragment of the gene encoding phosphoglycerate mutase protein; KV1000-F and KV1000-R that amplify a 438-bp fragment of the gene encoding thiopurine $\mathrm{S}$-methyltransferase protein and KV1615-F and KV1615-R that amplify a 499-bp fragment of the gene encoding $\mathrm{N}$-acetyltransferase protein (Table 1). Regarding the shared unique genes for the species $K$. pneumoniae, the following oligonucleotides were used: KP878-F and KP878-R that amplify an 878-bp fragment of the gene encoding transferase protein and KP888-F and KP888-R that amplify an 888bp fragment of the gene encoding phosphohydrolase protein (Table 1). Both the $m t n C$ gene and the shared unique genes of $K$. variicola and $K$. pneumoniae genomes, amplified by simple PCR, were highly specific to the respective bacterial genomes and Klebsiella genera (Figure 2A and B).

After the single-PCR amplification, the conditions for multiplex-PCR were determined. A mixture of $K$. variicola 801 and $K$. pneumoniae ATCC 13883 DNA was used as the positive control. The proposed multiplex PCR assays were as follows (Table 1): oligonucleotides KV770-F and -R, KP888-F and -R and KmtnC-F and -R, named M-PCR-1; oligonucleotides KV1615-F and -R, KP878-F and - R and KmtnC-F and -R, named M-PCR-2 and the oligonucleotides combination of KV1000-F and $-\mathrm{R}, \mathrm{KP} 888-\mathrm{F}$ and $-\mathrm{R}$ and $\mathrm{KmtnC}-\mathrm{F}$ and $-\mathrm{R}$, named $\mathrm{M}-$ PCR-3 (Figure 3A). Using the multiplex-PCR assays, all of the environmental endophytic $K$. variicola isolates [4] were identified correctly (Figure 3B). All of these experiments confirmed that the set of oligonucleotides of $K$. variicola and $K$. pneumoniae amplified only $K$. variicola and K. pneumoniae DNA, respectively.

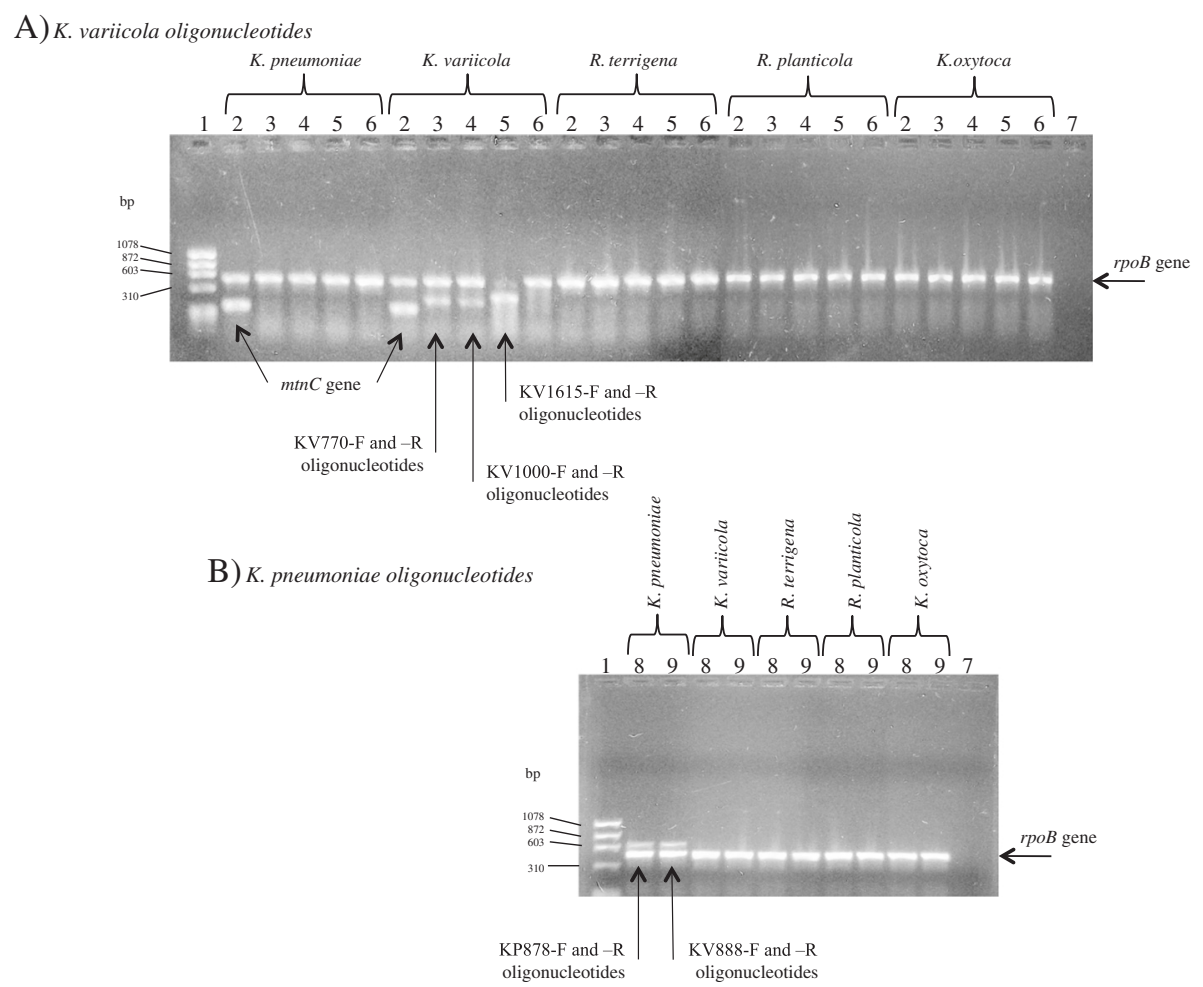

Figure 2 Amplification by PCR of shared unique genes to $K$. variicola and $K$. pneumoniae. A) Amplification of shared unique genes to $K$. variicola using the genomes of control strains such as K. pneumoniae ATCC 13883, K. variicola ATCC BAA-830 T, R. terrigena ATCC 33257 , R. platicola ATCC 33531 and K. oxytoca ATCC 49134. B) Amplification of shared unique genes to K. pneumoniae using the genomes of control strains described above. Lane 1, $\varphi$ X174/Hae III; Lane 2, mtnC gene (KmtnC-F and -R oligonucleotides); Lane 3, phosphoglycerate mutase gene (KV770-F and -R oligonucleotides); Lane 4, thiopurine S-methyltransferase gene (KV1000-F and -R oligonucleotides); Lane 5, N-acetyltransferase gene (KV1615-F and -R oligonucleotides); Lanes 1 to 6, rpoB gene (CM7 and rpoB-M) in combination with oligonucleotides of shared unique genes K. variicola; Lane 7, rpoB oligonuclotides without DNA (CM7 and rpoB-M); Lane 8, Transferase gene (KP878-F and R oligonucleotides); Lane 9, phosphohydrolase gene (KP888-F and R oligonucleotides) (Table 1). 


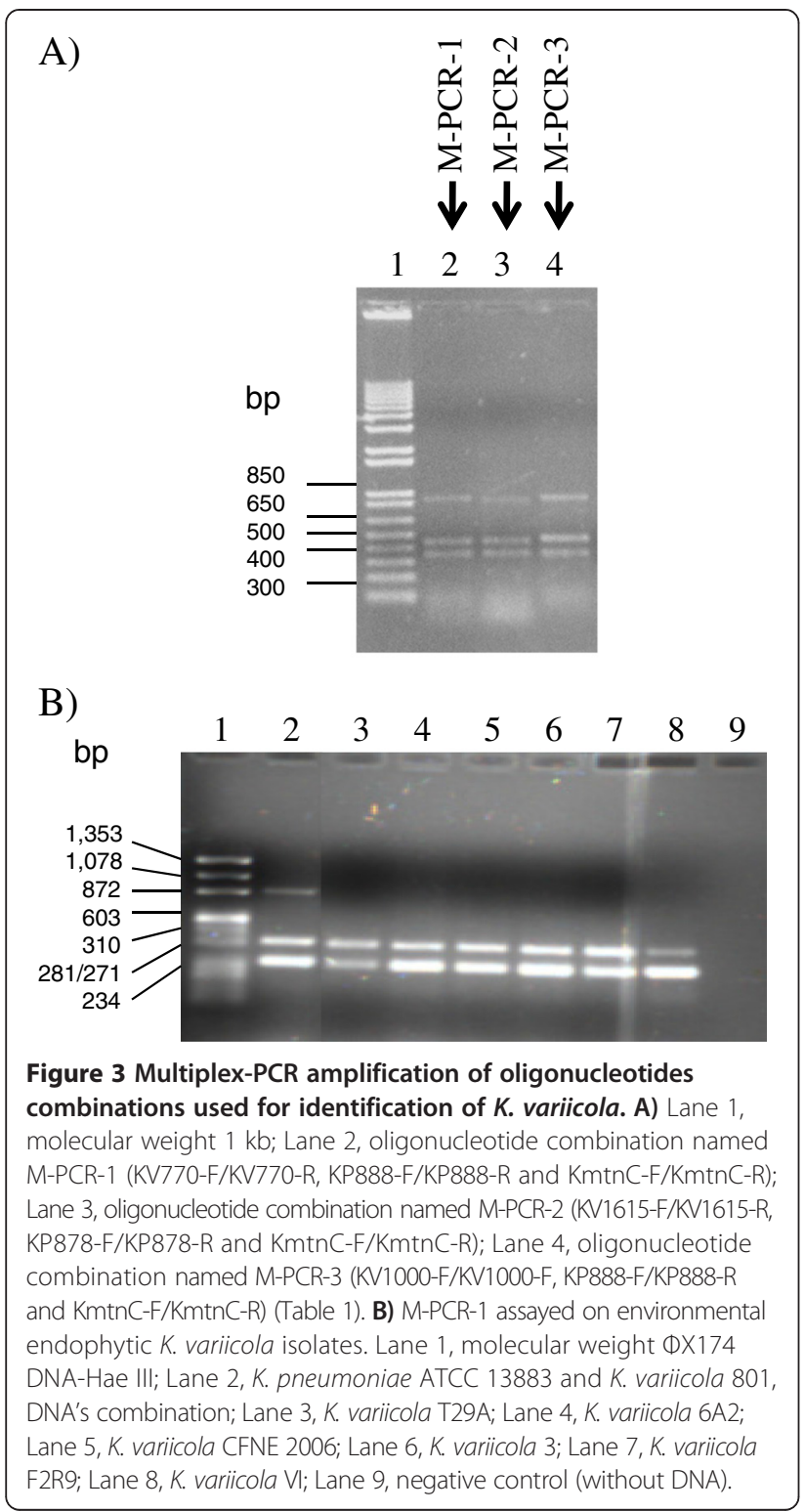

\section{Prevalence, susceptibility and characteristics of} K. variicola isolates

Finally, to evaluate our new multiplex PCR, we determined the prevalence of $K$. variicola isolates among a collection of $K$. pneumoniae isolates. The M-PCR-1 multiplex PCR assay was tested on a collection of 1,060 K. pneumoniae isolates (Additional file 1). The assay identified $23 \mathrm{~K}$. variicola isolates, which corresponded to a prevalence of $2.1 \%(23 / 1,060)$. The amount of $K$. variicola was smaller than that reported in previous studies; in 2004, two reports stated to have found $K$. variicola prevalences of $8 \%$ [4] and 11\%, respectively [24]. Considering that the phylogenetic group KpIII found by Brisse et al. [24] corresponded to $K$. variicola isolates, as reported by Rosentblueth et al. [4]. Of the remaining 1,034 K. variicola-negative isolates corresponded to $K$. pneumoniae bacterial species. Of them, $21 \mathrm{~K}$. pneumoniae clinical isolates were selected for the phylogenetic analysis of the $r p o B$ gene (see below). Of the $23(2.1 \%)$ K. variicolapositive isolates, $43.5 \%(10 / 23)$ were antimicrobial sensitive isolates. The antimicrobial susceptibility of these ten isolates was confirmed by disk diffusion against the main family of antimicrobials used in the hospital settings. These clinical isolates, together with the environmental $K$. variicola isolates, were sensitive to all of the antibiotics tested, except amoxicillin, with a negative-ESBL phenotype. Some clinical isolates showed an intermediate resistance to ticarcillin and nalidixic acid (Table 2). The multidrug-resistant $K$. variicola isolates showed a prevalence of $56.5 \%(13 / 23)$ and were ESBL producers (Table 2). These isolates showed resistance to amoxicillin, cephalothin, piperacillin, cefotaxime and ceftazidime, amikacin and ticarcillin. However, the isolates were sensitive to some $\beta$-lactam antibiotics (cefoxitin, cefuroxime and aztreonam) and to all quinolones and fluoroquinolones tested, as well as tetracycline (Table 2). With respect to ESBL genes, the ten isolates from the $K$. variicola outbreak identified in the hospital at Tabasco in 1996 showed SHV-2 [5] and SHV-2a ESBLs, with the latter being more prevalent (Table 2). In the other ESBL-producing $K$. variicola isolates, the ESBL SHV-5 and CTX-M-15 genes were identified, corresponding to isolates obtained at different hospitals, with multiple samples of origins and susceptibilities (Table 2).

One relevant finding of this study is that $K$. variicola isolates could be both multidrug resistant and sensitive. Given that $K$. variicola clinical isolates were obtained from patients where $K$. pneumoniae could also be isolated. The assumption that $K$. variicola could coexist in the same patient with $K$. pneumoniae was recently determined [28]. This notion reveals the possibility of $K$. variicola being able to acquire resistance genes to $\beta$-lactam and/or other antibiotics from some other bacteria, as could be the case of the ESBL SHV-type and CTX-M-15 genes identified in this study. The first family has been described in K. pneumoniae isolates; in fact, the CTX-M family is the most prevalent both in Mexico [16] and worldwide [29]. Previous studies have reported that isolates from the KpIII phylogenetic group displayed resistance to ampicillin, carbenicillin, piperacillin, gentamicin, ceftazidime, and ceftriaxone [24]. The resistance to penicillin antibiotics is in agreement with the LEN-type $\beta$-lactamases identified in the phylogenetic group KpIII [30]. In the present study, all $K$. variicola isolates studied were at least amoxicillin resistant (Table 2), most likely due to a chromosomal LENtype $\beta$-lactamase. 
Table 2 Characteristics of endophytic and clinical $K$. variicola isolates

\begin{tabular}{|c|c|c|c|c|c|c|c|c|c|c|c|c|c|c|c|c|c|c|c|c|c|c|}
\hline \multirow[t]{2}{*}{ Isolate } & \multirow[t]{2}{*}{ Hospital $^{\mathrm{a}}$} & \multirow{2}{*}{$\begin{array}{l}\text { Isolation } \\
\text { date }\end{array}$} & \multirow{2}{*}{$\begin{array}{l}\text { Origin of } \\
\text { the sample }\end{array}$} & \multirow{2}{*}{$\begin{array}{l}\text { PFGE } \\
\text { pattern }\end{array}$} & \multirow{2}{*}{$\begin{array}{l}\text { ESBL } \\
\text { production }\end{array}$} & \multirow[t]{2}{*}{ ESBL } & \multicolumn{16}{|c|}{ Antimicrobial susceptibility ${ }^{\mathbf{b}}$} \\
\hline & & & & & & & AMX & $\mathrm{CF}$ & FOX & CXM & PIP & TZP & ATM & CTX & CAZ & GM & AMK & TIC & TET & NAL & CIP & LUX \\
\hline 1109 & 1 & 1996 & Blood & NR & - & Negative & $\mathrm{R}$ & S & s & S & s & s & R & R & $\mathbf{R}$ & $\mathbf{R}$ & $\mathbf{R}$ & I & S & s & S & S \\
\hline 801 & 2 & 1996 & Blood & A & + & SHV-2a & $\mathrm{R}$ & $\mathbf{R}$ & s & S & $\mathbf{R}$ & s & s & $\mathrm{R}$ & $\mathbf{R}$ & $\mathbf{R}$ & $\mathbf{R}$ & $\mathrm{R}$ & S & s & S & S \\
\hline 803 & 2 & 1996 & Blood & A & + & SHV-2a & R & $\mathbf{R}$ & s & । & $\mathbf{R}$ & s & s & $\mathbf{R}$ & 1 & S & $\mathrm{R}$ & $\mathrm{R}$ & $S$ & s & S & S \\
\hline 804 & 2 & 1996 & Blood & A & + & SHV-2a & $\mathrm{R}$ & $\mathrm{R}$ & S & S & $\mathbf{R}$ & s & s & $\mathrm{R}$ & 1 & S & $\mathrm{R}$ & $\mathrm{R}$ & S & S & S & S \\
\hline 805 & 2 & 1996 & Blood & A & + & SHV-2a & $\mathrm{R}$ & $\mathrm{R}$ & s & S & $\mathbf{R}$ & s & s & $\mathrm{R}$ & 1 & S & $\mathrm{R}$ & $\mathrm{R}$ & S & S & S & S \\
\hline 806 & 2 & 1996 & Blood & A & + & SHV-2 & $\mathrm{R}$ & $\mathrm{R}$ & S & S & $\mathbf{R}$ & s & S & $\mathrm{R}$ & 1 & S & $\mathrm{R}$ & $\mathrm{R}$ & S & S & S & S \\
\hline 807 & 2 & 1996 & Blood & A & + & SHV-2a & $\mathrm{R}$ & $\mathrm{R}$ & s & S & $\mathbf{R}$ & S & S & $\mathrm{R}$ & $\mathrm{R}$ & S & $\mathrm{R}$ & $\mathrm{R}$ & S & S & S & S \\
\hline 808 & 2 & 1996 & Blood & A & + & SHV-2a & $\mathrm{R}$ & $\mathrm{R}$ & $\mathrm{R}$ & S & $\mathbf{R}$ & S & S & $\mathrm{R}$ & 1 & 1 & $\mathrm{R}$ & $\mathrm{R}$ & S & S & S & S \\
\hline 809 & 2 & 1996 & Blood & A & + & SHV-2a & $\mathrm{R}$ & $\mathrm{R}$ & $\mathbf{R}$ & S & $\mathbf{R}$ & S & 1 & $\mathrm{R}$ & $\mathrm{R}$ & $\mathrm{R}$ & $\mathrm{R}$ & $\mathrm{R}$ & S & S & S & S \\
\hline 811 & 2 & 1996 & Blood & A & + & SHV-2a & $\mathrm{R}$ & $\mathrm{R}$ & $\mathrm{R}$ & S & $\mathbf{R}$ & S & S & $\mathrm{R}$ & $\mathrm{R}$ & 1 & $\mathrm{R}$ & $\mathrm{R}$ & S & S & S & S \\
\hline 812 & 2 & 1996 & Blood & A & + & SHV-2a & $\mathrm{R}$ & $\mathrm{R}$ & $\mathrm{R}$ & S & $\mathbf{R}$ & S & S & $\mathrm{R}$ & $\mathrm{R}$ & I & $\mathrm{R}$ & $\mathrm{R}$ & S & S & S & S \\
\hline 1171 & 1 & 1998 & Catheter & NR & - & Negative & $\mathrm{R}$ & S & S & S & S & S & S & S & S & S & S & I & S & S & S & S \\
\hline 1258 & 3 & 1999 & Blood & NR & + & SHV-5 & $\mathrm{R}$ & $\mathbf{R}$ & $\mathrm{R}$ & I & $\mathbf{R}$ & s & $\mathbf{R}$ & S & s & $\mathbf{R}$ & $\mathrm{R}$ & $\mathrm{R}$ & S & s & s & S \\
\hline $06-268$ & 4 & 2007 & Abscess & NR & + & СТХ M- 15 & $\mathrm{R}$ & $\mathbf{R}$ & s & S & s & s & $\mathbf{R}$ & $\mathrm{R}$ & $\mathrm{R}$ & S & S & 1 & S & s & S & S \\
\hline 8917 & 5 & 2011 & sputum & NR & - & Negative & $\mathrm{R}$ & S & s & S & s & s & s & S & s & S & S & S & S & s & S & S \\
\hline 9635 & 6 & 2011 & Catheter & NR & - & Negative & $\mathrm{R}$ & S & s & S & s & s & s & $\mathrm{R}$ & $\mathrm{R}$ & S & S & 1 & S & s & S & S \\
\hline 9351 & 6 & 2012 & Secretion & NR & - & Negative & $\mathrm{R}$ & S & S & S & S & S & S & S & S & S & S & 1 & S & 1 & S & S \\
\hline 9352 & 6 & 2012 & Secretion & NR & - & Negative & $\mathrm{R}$ & S & s & S & s & s & s & S & S & S & S & S & S & S & S & S \\
\hline 9353 & 6 & 2012 & Biliary liquids & NR & - & Negative & $\mathrm{R}$ & S & S & S & S & S & S & S & S & S & S & 1 & S & S & S & S \\
\hline 4880 & 6 & 2013 & Blood & NR & - & Negative & $\mathrm{R}$ & S & S & S & S & S & S & S & S & S & S & 1 & S & 1 & S & S \\
\hline 9326 & 6 & 2013 & Secretion & NR & - & Negative & $\mathrm{R}$ & S & S & S & S & S & S & S & S & S & S & S & S & S & S & S \\
\hline 9387 & 6 & 2013 & Secretion & NR & - & Negative & $\mathrm{R}$ & S & s & s & s & s & s & s & s & s & s & 1 & s & s & s & s \\
\hline 9388 & 6 & 2013 & Urine & NR & - & Negative & $\mathrm{R}$ & S & s & S & s & s & s & S & S & S & S & I & S & s & S & S \\
\hline 9925 & 7 & 2013 & Abscess & NR & - & Negative & $\mathrm{R}$ & S & s & S & s & s & s & S & S & S & S & I & S & s & S & s \\
\hline F2R9 & NA & 2003 & Banana root & NR & - & Negative & $\mathrm{R}$ & S & S & S & S & S & S & S & S & S & S & S & S & S & S & S \\
\hline T29A & NA & 2003 & Sugar cane stem & NR & & Negative & $\mathbf{R}$ & S & S & S & S & S & S & S & S & S & S & S & S & S & S & S \\
\hline $6 \mathrm{~A} 2$ & NA & 2003 & Banana leaves & NR & - & Negative & R & S & S & S & S & S & S & S & S & S & S & S & S & S & S & S \\
\hline $\mathrm{Vl}$ & NA & 2003 & Banana stem & B & - & Negative & $\mathrm{R}$ & S & S & S & S & s & S & S & S & S & S & S & S & S & S & S \\
\hline 3 & NA & 2003 & Maize shoots & B & - & Negative & $\mathrm{R}$ & S & S & S & S & S & S & S & S & S & S & S & S & S & S & S \\
\hline CFNE-2006 & NA & 2003 & Rice roots & NR & - & Negative & $\mathrm{R}$ & s & S & S & s & s & s & S & S & S & S & S & S & s & S & S \\
\hline
\end{tabular}

a Hospitlas: 1, Hospital de Niño y del Adolesente Morelense (HNAM); 2, Hospital del Niño de Tabasco (HNT);i 3, Hospital Infantil de Mexico (HIM); 4, Centro Regional de Control de Enfermedades Infecciosas (CRCEI); 5 , Clinica del ISSSTE-Morelos (CHM); 6, Hospital Civil de Guadalajara (HCG); 7, Hospital Manuel Gea Gonzalez (HMGG).

Abbreviations: Amoxicillin, AMX; Cephalothin, CF; Cefoxitin, FOX; Cefuroxime, CXM; Piperaciline, PIP; Piperaciline/Tazobactam, TZP; Aztreonam, ATM; Cefotaxime, CTX; Ceftazidime, CAZ, Gentamicin, GM; Amikacin, AMK; Ticarcillin, TIC; Tetracycline, TET; Nalidixic Acid, NAL; Ciprofloxacine, CIP; Levofloxacin, LUX. 


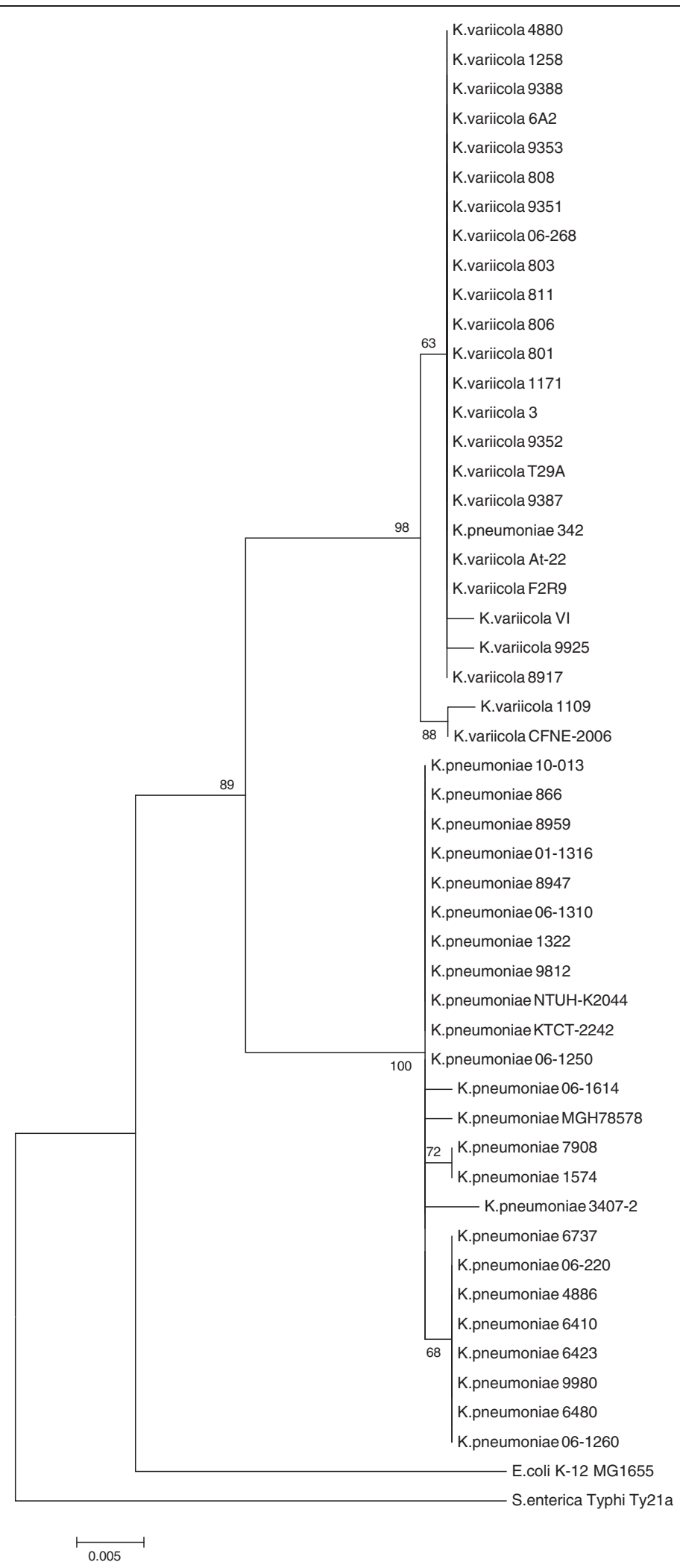

Figure 4 The maximum-likelihood phylogeny of the $r p o B$ sequences. The tree was rooted with the sequences from Escherichia coli K-12 MG1655 and Salmonella enterica Ty21. To evaluate the support of the nodes, a bootstrap analysis of 100 replicates was conducted. For clarity, only the bootstrap values for the main groups are shown. The scale bar represents substitutions per site. 
$r p o B$ analysis and fingerprinting of the $K$. variicola isolates identified

The phylogeny of $r p o B$ gene sequences of $K$. variicola and $K$. pneumoniae isolates clearly shows two strongly supported clusters (Figure 4); one corresponds to $K$. variicola isolates plus $K$. pneumoniae 342, whereas the other comprises $K$. pneumoniae isolates. In accordance with the approach (Figure 1), the phylogeny of rpoB sequences supports the differentiation of these related bacterial species. The relationship of $K$. variicola of clinical and environmental isolates was investigated by PFGE. The analysis showed two clonal groups (Figure 5 and Table 2). Clone A corresponds to $K$. variicola outbreak isolates from a pediatric hospital, whereas clone B cluster corresponds to two environmental isolates collected from maize and banana obtained from different cities (Figure 5). However, the fingerprinting analysis observed among $K$. variicola and $K$. pneumoniae isolates is not correlated with the bacterial species (Figure 5).

The multiplex PCR proposed in this study is an effective tool to differentiate $K$. variicola from $K$. pneumoniae because $100 \%$ of the clinical isolates were correctly identified, as were the control strains included. The reliability of the assay proposed was corroborated through the phylogenetic analysis of the rpoB gene from $K$. variicola and $K$. pneumoniae isolates identified previously by multiplex PCR. The sequence analysis of the $\operatorname{rpoB}$ gene was proven to be a useful tool for bacterial classification, particularly for Enterobacteriaceae species [31] and $K$. variicola [4]. The few shared unique genes to $K$. variicola and $K$. pneumoniae used in this study by multiplex PCR did not correspond to unique specific genes for each species. Instead, they corresponded to unique genes that clearly match with the $K$. variicola and $K$. pneumoniae

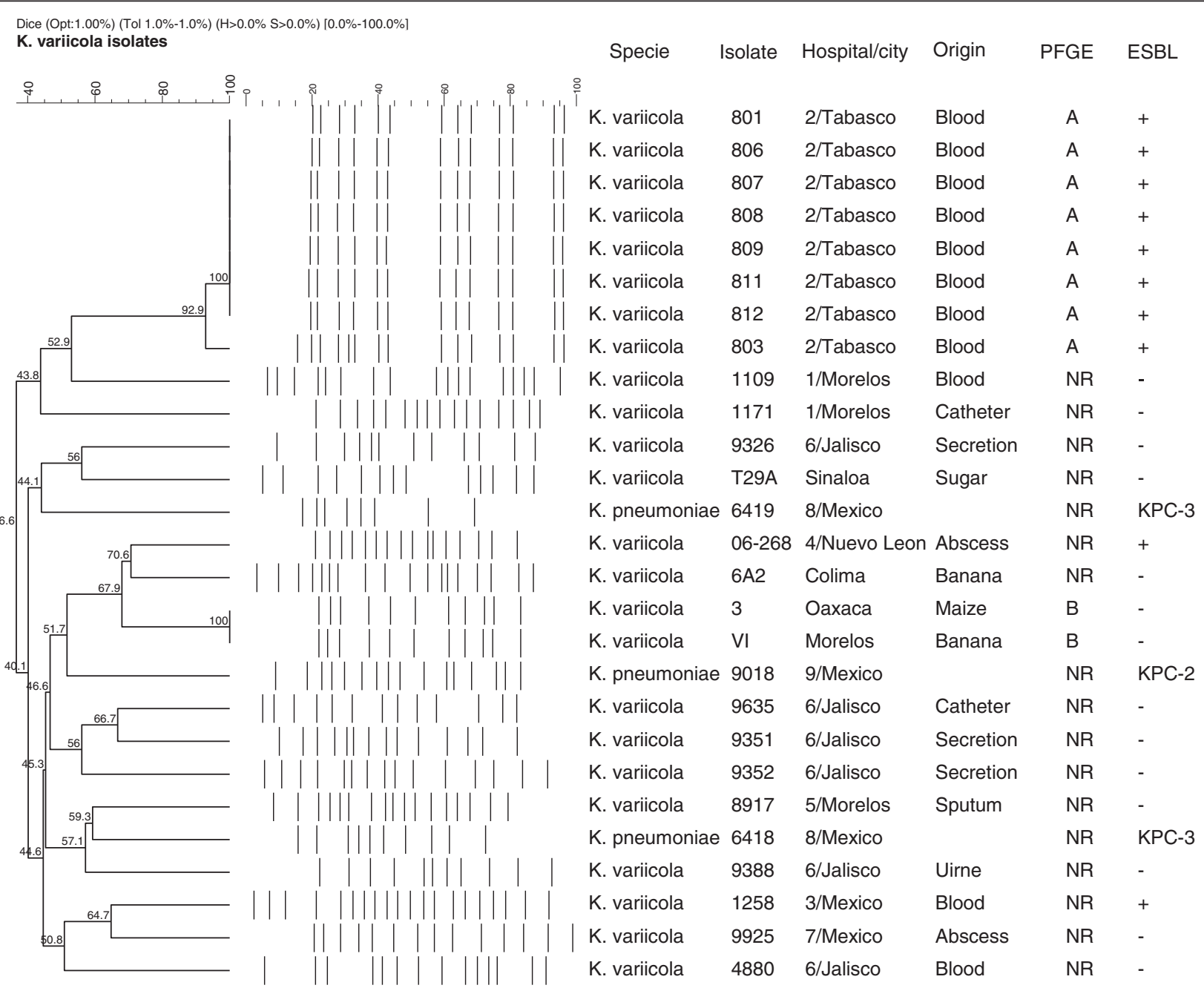

Figure 5 PFGE and dendrogram analysis that includes $\boldsymbol{K}$. variicola and $\boldsymbol{K}$. pneumoniae isolates. The figure shows the isolate (environmental and clinical), species, hospital and city, origin of sample, PFGE pattern and ESBL-producer phenotype. 
genomes. The selected genes encode proteins that are involved in cellular metabolism in the case of $K$. variicola. We note that the shared unique genes of $K$. variicola analyzed by a recent BLASTn search have matches with a recently submitted $K$. pneumoniae KP5-1 genome, an isolate obtained from a known cotton boll pest. The housekeeping gene analysis of this K. pneumoniae KP5-1 isolate was compared with the housekeeping genes of $K$. variicola and $K$. pneumoniae genomes included in this study. The results showed that the K. pneumoniae KP5-1 genome fell within the $K$. variicola monophyletic group (Figure 1) and the chromosomal LEN-16 $\beta$-lactamase gene (Additional file 2). We considered that this bacterial genome corresponds to other $K$. variicola genomes deposited in GenBank database and is called a K. pneumoniae species.

\section{Conclusions}

$K$. variicola and $K$. pneumoniae are closely related sister species that share many phenotypic properties; thus, $K$. variicola has been mistaken for $K$. pneumoniae for a long time and around the world. Our multiplex PCR provides the means to properly identify and genotype $K$. variicola and, therefore, this tool could be very helpful for clinical, epidemiological, population genetics and environmental studies of this species. For example, two recent reports have described $K$. variicola as a frequent cause of bloodstream infection, as being associated with higher mortality than K. pneumoniae [32], and as being associated with clinical bovine mastitis [33]. In addition, we determined that a previous collection of K. pneumoniae isolates has a low but significant prevalence of $K$. variicola isolates. We established that both multidrugresistant and multidrug-sensitive isolates could colonize humans. We believe that our multiplex-PCR assay could be of paramount importance to understanding the population dynamics of $K$. variicola in both clinical and environmental settings.

\section{Additional files}

\section{Additional file 1: Clinical isolates of $K$. pneumoniae included in the} study.

Additional file 2: The maximum likelihood phylogeny of LEN-alleles amino acid sequences. To evaluate the support of the nodes, a bootstrap analysis of 100 replicates was conducted and using the Jones-Taylor-Thornton substitution model. The scale bar represents substitutions per site.

Additional file 3: Shared genes identified both $K$. variicola as $K$. pneumoniae.

Additional file 4: Metabolic and structural genes both $K$. variicola as K. pneumoniae.

\section{Competing interests}

The authors declare that they have no competing interests.

\section{Authors' contributions}

EMR and JSS made substantial contributions to the acquisition and interpretation of the bacterial isolates. PT and MPG carried out the multiplex-PCR assays and $K$. variicola screening. $\mathrm{HB}$ and $\mathrm{JMB}$ conducted the bioinformatic analysis of the $K$. pneumoniae and $K$. variicola genomes. REGB and JT sequenced and assembled the K. variicola 801 genome. UGR coordinated and designed the study. UGR wrote the manuscript. All authors read and approved the final manuscript.

\section{Acknowledgements}

This work was funded by Consejo Nacional de Ciencia y Tecnología (CONACyT), SEP-CONACYT grant number 130224. We thank Sánchez-Pérez A. for her excellent laboratory assistance.

\section{Author details}

'Departamento de Diagnóstico Epidemiológico, Av. Universidad \# 655, Col. Sta. Ma. Ahuacatitlán, C.P. 62100 Cuernavaca, Morelos, Mexico. ${ }^{2}$ Centro de Ciencias Genómicas (CCG), Universidad Nacional Autónoma de México (UNAM), Cuernavaca, Morelos, Mexico. ${ }^{3}$ Instituto Nacional de Salud Pública (INSP), Centro de Investigación Sobre Enfermedades Infecciosas (CISEI), Departamento de Inmunología, Cuernavaca, México.

Received: 19 August 2014 Accepted: 20 February 2015

Published online: 13 March 2015

\section{References}

1. Podschun R, Ullmann U. Klebsiella spp. as nosocomial pathogens: epidemiology, taxonomy, typing methods, and pathogenicity factors. Clin Microbiol Rev. 1998;11:589-603

2. Pinto-Tomas AA, Anderson MA, Suen G, Stevenson DM, Chu FS, Cleland $W W$, et al. Symbiotic nitrogen fixation in the fungus gardens of leaf-cutter ants. Science. 2009;326:1120-3.

3. Fouts DE, Tyler HL, DeBoy RT, Daugherty S, Ren Q, Badger JH, et al. Complete genome sequence of the N2-fixing broad host range endophyte Klebsiella pneumoniae 342 and virulence predictions verified in mice. PLoS Genet. 2008;4:e1000141.

4. Rosenblueth M, Martinez L, Silva J, Martinez-Romero E. Klebsiella variicola, a novel species with clinical and plant-associated isolates. Syst Appl Microbiol. 2004:27:27-35

5. Garza-Ramos U, Martinez-Romero E, Silva-Sanchez J. SHV-type extended-spectrum beta-lactamase (ESBL) are encoded in related plasmids from enterobacteria clinical isolates from Mexico. Salud Publica Mex. 2007;49:415-21

6. Westbrook GL, O'Hara CM, Roman SB, Miller JM. Incidence and identification of Klebsiella planticola in clinical isolates with emphasis on newborns. J Clin Microbiol. 2000;38:1495-7.

7. Alves MS, Dias RC, de Castro AC, Riley LW, Moreira BM. Identification of clinical isolates of indole-positive and indole-negative Klebsiella spp. J Clin Microbiol. 2006:44:3640-6.

8. Hazen TH, Zhao L, Sahl JW, Robinson G, Harris AD, Rasko DA, et al. Characterization of Klebsiella sp. 10982, a colonizer of humans that contains novel antibiotic resistance alleles and exhibits genetic similarities to plant and clinical Klebsiella isolates. Antimicrob Agents Chemother. 2014;58:1879-88.

9. Kumar V, Sun P, Vamathevan J, Li Y, Ingraham K, Palmer L, et al. Comparative genomics of Klebsiella pneumoniae strains with different antibiotic resistance profiles. Antimicrob Agents Chemother. 2011;55:4267-76.

10. Tamura K, Peterson D, Peterson N, Stecher G, Nei M, Kumar S. MEGA5: molecular evolutionary genetics analysis using maximum likelihood, evolutionary distance, and maximum parsimony methods. Mol Biol Evol. 2011;28:2731-9.

11. Altschul SF, Gish W, Miller W, Myers EW, Lipman DJ. Basic local alignment search tool. J Mol Biol. 1990;215:403-10.

12. Sekowska A, Denervaud V, Ashida H, Michoud K, Haas D, Yokota A, et al. Bacterial variations on the methionine salvage pathway. BMC Microbiol. 2004;4:9

13. Wu KM, Li LH, Yan Jل, Tsao N, Liao TL, Tsai HC et al. Genome sequencing and comparative analysis of Klebsiella pneumoniae NTUH-K2044, a strain causing liver abscess and meningitis. J Bacteriol. 2009:191:4492-501.

14. Diancourt L, Passet V, Verhoef J, Grimont PA, Brisse S. Multilocus sequence typing of Klebsiella pneumoniae nosocomial isolates. J Clin Microbiol. 2005;43:4178-82 
15. Clinical and Laboratory Standards Institute. Methods for Dilution Antimicrobial Susceptibility Test for Bacteria that grown aerobically; Approved Standard. M07-A9, vol. 32[2]. 2012.

16. Silva-Sanchez J, Barrios H, Reyna-Flores F, Bello-Diaz M, Sanchez-Perez A, Rojas T, et al. Prevalence and characterization of plasmid-mediated quinolone resistance genes in extended-spectrum beta-lactamase-producing Enterobacteriaceae isolates in Mexico. Microb Drug Resist. 2011;17:497-505.

17. Tenover FC, Arbeit RD, Goering RV, Mickelsen PA, Murray BE, Persing DH, et al. Interpreting chromosomal DNA restriction patterns produced by pulsed-field gel electrophoresis: criteria for bacterial strain typing. J Clin Microbiol. 1995;33:2233-9.

18. Garza-Ramos U, Barrios H, Reyna-Flores F, Sanchez-Perez A, Tamayo-Legorreta E, Ibarra-Pacheco A, et al. Characteristics of KPC-2-producing Klebsiella pneumoniae (ST258) clinical isolates from outbreaks in 2 Mexican medical centers. Diagn Microbiol Infect Dis. 2014;79:483-5.

19. Rodriguez-Zulueta P, Silva-Sanchez J, Barrios H, Reyes-Mar J, Velez-Perez F, Arroyo-Escalante S, et al. First outbreak of KPC-3-producing Klebsiella pneumoniae (ST258) clinical isolates in a Mexican Medical Center. Antimicrob Agents Chemother. 2013;57:4086-8.

20. Ramos PI, Picao RC, Almeida LG, Lima NC, Girardello R, Vivan AC, et al. Comparative analysis of the complete genome of KPC-2-producing Klebsiella pneumoniae Kp13 reveals remarkable genome plasticity and a wide repertoire of virulence and resistance mechanisms. BMC Genomics. 2014;15:54.

21. Andrade BG, de Veiga RN, Abanto Marin MF, Fonseca EL, Vicente AC. The genome of a clinical $\mathrm{K}$. variicola strain reveals virulence associated traits and a pl9-like plasmid. FEMS Microbiol Lett. 2014:360:13-6.

22. Rubio LM, Ludden PW. Biosynthesis of the iron-molybdenum cofactor of nitrogenase. Annu Rev Microbiol. 2008:62:93-111.

23. Arnold W, Rump A, Klipp W, Priefer UB, Puhler A. Nucleotide sequence of a 24,206-base-pair DNA fragment carrying the entire nitrogen fixation gene cluster of Klebsiella pneumoniae. J Mol Biol. 1988;203:715-38.

24. Brisse $S$, Van Himbergen T, Kusters K, Verhoef J. Development of a rapid identification method for Klebsiella pneumoniae phylogenetic groups and analysis of 420 clinical isolates. Clin Microbiol Infect. 2004;10:942-5.

25. van Veen SQ, Claas EC, Kuijper EJ. High-throughput identification of bacteria and yeast by matrix-assisted laser desorption ionization-time of flight mass spectrometry in conventional medical microbiology laboratories. J Clin Microbiol. 2010;48:900-7.

26. Soumet C, Ermel G, Rose V, Rose N, Drouin P, Salvat G, et al. Identification by a multiplex PCR-based assay of Salmonella typhimurium and Salmonella enteritidis strains from environmental swabs of poultry houses. Lett Appl Microbiol. 1999;29:1-6.

27. Soumet C, Ermel G, Rose N, Rose V, Drouin P, Salvat G, et al. Evaluation of a multiplex PCR assay for simultaneous identification of Salmonella sp., Salmonella enteritidis and Salmonella typhimurium from environmental swabs of poultry houses. Lett Appl Microbiol. 1999;28:113-7.

28. Garza-Ramos U, Moreno-Dominguez S, Hernández-Castro R, Barrios H, Reyna-Flores F, Sanchez-Perez A, et al. A co-infection of SHV-12-KPC-3producing ST258 K. pneumoniae and susceptible-K. variicola clinical isolates. 2015. Under review.

29. Bonnet R. Growing group of extended-spectrum beta-lactamases: the CTX-M enzymes. Antimicrob Agents Chemother. 2004:48:1-14.

30. Haeggman S, Lofdahl S, Paauw A, Verhoef J, Brisse S. Diversity and evolution of the class A chromosomal beta-lactamase gene in Klebsiella pneumoniae. Antimicrob Agents Chemother. 2004;48:2400-8.

31. Mollet C, Drancourt M, Raoult D. rpoB sequence analysis as a novel basis for bacterial identification. Mol Microbiol. 1997:26:1005-11.

32. Maatallah M, Vading M, Kabir MH, Bakhrouf A, Kalin M, Naucler P, et al. Klebsiella variicola Is a Frequent Cause of Bloodstream Infection in the Stockholm Area, and Associated with Higher Mortality Compared to K. pneumoniae. PLoS One. 2014;9:e113539.

33. Podder MP, Rogers L, Daley PK, Keefe GP, Whitney HG, Tahlan K. Klebsiella species associated with bovine mastitis in Newfoundland. PLoS One. 2014:9:e106518.

\section{Submit your next manuscript to BioMed Central and take full advantage of:}

- Convenient online submission

- Thorough peer review

- No space constraints or color figure charges

- Immediate publication on acceptance

- Inclusion in PubMed, CAS, Scopus and Google Scholar

- Research which is freely available for redistribution 